\title{
BMJ Open Measuring patient-centred system performance: a scoping review of patient-centred care quality indicators
}

Maria-Jose Santana, ${ }^{1,2}$ Sadia Ahmed, ${ }^{2}$ Diane Lorenzetti, ${ }^{1,3}$ Rachel J Jolley, ${ }^{1}$ Kimberly Manalili, ${ }^{1}$ Sandra Zelinsky, ${ }^{1}$ Hude Quan, ${ }^{1}$ Mingshan Lu ${ }^{1,4}$

To cite: Santana M-J, Ahmed S, Lorenzetti D, et al. Measuring patient-centred system performance: a scoping review of patient-centred care quality indicators. BMJ Open 2019;9:e023596. doi:10.1136/ bmjopen-2018-023596

- Prepublication history and additional material for this paper are available online. To view these files, please visit the journal online (http://dx.doi. org/10.1136/bmjopen-2018023596).

Received 13 April 2018

Revised 15 November 2018

Accepted 16 November 2018

Check for updates

(c) Author(s) (or their employer(s)) 2019. Re-use permitted under CC BY-NC. No commercial re-use. See rights and permissions. Published by BMJ.

${ }^{1}$ Community Health Sciences, University of Calgary, Calgary, Alberta, Canada

${ }^{2}$ Department of Pediatrics, Cumming School of Medicine, University of Calgary, Calgary, Alberta, Canada

${ }^{3}$ Health Sciences Library, University of Calgary, Calgary, Alberta, Canada

${ }^{4}$ Department of Economics, University of Calgary, Calgary, Alberta, Canada

Correspondence to Dr Maria-Jose Santana; mjsantan@ucalgary.ca

\section{ABSTRACT}

Objectives The shift to the patient-centred care (PCC) model as a healthcare delivery paradigm calls for systematic measurement and evaluation. In an attempt to develop patient-centred quality indicators (PC-QIs), this study aimed to identify quality indicators that can be used to measure PCC.

Methods Design: scoping review. Data Sources: studies were identified through searching seven electronic databases and the grey literature. Search terms included quality improvement, quality indicators, healthcare quality and PCC. Eligibility Criteria: articles were included if they mentioned development and/or implementation of PC-Qls. Data Extraction and Synthesis: extracted data included study characteristics (country, year of publication and type of study/article), patients' inclusion in the development of indicators and type of patient populations and point of care if applicable (eg, in-patient, out-patient and primary care). Results A total 184 full-text peer-reviewed articles were assessed for eligibility for inclusion; of these, 9 articles were included in this review. From the non-peer-reviewed literature, eight documents met the criteria for inclusion in this study. This review revealed the heterogeneity describing and defining the nature of PC-Qls. Most PCQls were presented as PCC measures and identified as guidelines, surveys or recommendations, and therefore cannot be classified as actual PC-Qls. Out of 502 ways to measure PCC, only 25 were considered to be actual PCQls. None of the identified articles implemented the quality indicators in care settings.

Conclusion The identification of PC-Qls is a key first step in laying the groundwork to develop evidence-based PC-Qls. Research is needed to continue the development and implementation of PC-Qls for healthcare quality improvement.

\section{INTRODUCTION}

Patient-centred care (PCC) is one of the six dimensions of healthcare and was formally described by the Institute of Medicine in 2001 as healthcare that respects and responds to the preferences, needs and values of the individual patients throughout all healthcare decisions. ${ }^{1}$ PCC is an approach that has become central to policies and programming to improve healthcare efficiencies and address patient safety issues. ${ }^{2}$

\section{Strengths and limitation of this study}

- Transparent and rigorous search strategy.

- Involvement of patient partner in the study.

- Study informed by a previously published protocol.

- Search strategy using only English terms.

- We did not assess the quality of the measures and/ or indicators identified.

PCC is a model in which healthcare providers are encouraged to partner with patients and families to design and deliver individualised care. PCC models have been linked to positive patient experiences and improved outcomes, such as increased adherence to care and treatment. ${ }^{3-5}$ In the literature, a PCC approach has been found to benefit patients and healthcare organisations in reducing costs, for instance through decreasing the length of hospital stays and readmission rates. ${ }^{6}{ }^{7}$ However, PCG is conceptualised differently among different stakeholders, impacting effective implementation in care settings. ${ }^{8}$ The adoption of a PCG model requires first, the identification of appropriate indicators to measure the quality of PCC, and second, the assessment of the impact of delivering PCC on healthcare system and patient outcomes. ${ }^{9}$

Quality indicators are tools that measure system performance and healthcare quality, and demonstrate the extent to which improvement efforts have led to desirable change, or contributed to unintended results. ${ }^{10}$ As identified from the Agency for Healthcare Research and Quality (AHRQ), a quality indicator consists of a specific aspect of quality being captured, and a method for how concepts of quality are captured (which includes data source, measure type, observable event, specification and risk adjustment). ${ }^{11}$ While various quality indicators have been developed to measure healthcare safety, effectiveness and access, ${ }^{12}$ they typically do 
not incorporate the priorities and experiences of patients and family caregivers. To truly evaluate the impact of patient-centredness, indicators must reflect the patient and family caregiver perspective.

This scoping review aimed to synthesise existing literature on quality indicators used in the evaluation of PCC. This review was guided by the questions: 'What PC-QIs have been developed to measure patient-centred care?' 'How are patient-centred quality indicators defined?' and 'Have existing PC-QIs been implemented and evaluated across various points-of-care settings, processes of care and at the systems level to measure patient-centred care?' The information gained from this study will inform the development of PCC quality indicators that could be implemented to drive healthcare improvement valued by patients and families.

\section{METHODS}

We employed a scoping review protocol that was previously published, ${ }^{13}$ using methodology based on Arksey and O'Malley's scoping review framework ${ }^{14}$ and Levac et $a l$ 's methodological enhancement. ${ }^{15}$ We searched the peer-reviewed published and grey literature for either proposed or existing quality indicators that have been developed and/or implemented across various points-of-care settings to measure PCC. For this scoping review, the $A H R Q^{16}$ definition of a quality indicator was adapted to incorporate a patient and family focus. Specifically, a PC-QI was defined as the unit of measurement of healthcare system, organisational or individual performance, that quantifies patients' and families' experiences with the care received and the experience of any individual who needs to contact with healthcare services. In contrast, PCC measures are in the form of a survey, guideline or recommendation.

\section{Data sources and search strategy}

In order to identify studies assessing quality indicators for PCC, search strategies were developed that combined terms from two concepts: PCG and PC-QIs (online supplementary file 1). The Cochrane Library, the Cumulative Index to Nursing and Allied Health Literature (CINAHL), EMBASE, MEDLINE, PsycINFO, Social Services Abstracts and Social Work Abstracts were searched from inception to May 2017. A search of the Google search engines, and the websites of relevant quality improvement and patient-centric organisations enabled the identification of relevant grey literature. Grey literature searches were conducted in duplicate by both a researcher and patient partner. The reference lists of included studies were also scanned to identify any other studies of relevance.

\section{Citation management}

All references were imported into a custom-written Java software application, Synthesis for reference management and data collection. ${ }^{17}$ Duplicate citations were removed automatically by the software, with any mismatched duplicates removed manually if detected.

\section{Study selection and data abstraction}

To be eligible for inclusion, the study/article had to (1) identify quality indicators for PCC and/or (2) identify PC-QI in performance measurement (eg, validation). The title and abstract of each citation identified was screened for eligibility independently by two reviewers (M-JS and ML). The full text of any abstract selected by either reviewer was retrieved and assessed for eligibility. Any full-text articles for which there was inter-rater discord were reviewed a second time, and final disagreements about study eligibility were resolved through discussion.

\section{Data collection and classification of indicators}

A data collection tool was developed and tested on a sample of papers to determine its practicality prior to the full review (online supplementary file 2). Extracted data included study characteristics (country, year of publication and type of study/article), patients' inclusion in the development of indicators and type of patient populations and point of care if applicable (eg, in-patient, out-patient and primary care).

All extracted indicators were classified collaboratively by two authors (M-JS and SA) according to a person-centred care framework ${ }^{18}$ developed by the team and guided by the Donabedian model of quality of care. ${ }^{19}$ This framework provides a roadmap for healthcare systems to implement and measure PCC at the level of structure (the healthcare system/organisational level), process (the patient-healthcare provider interaction level) and outcome (the patient-health care provider and healthcare systems interaction level). ${ }^{18}$

\section{Patient involvement}

Levac et al recommend the involvement of stakeholders in the scoping review methodology. ${ }^{15}$ We worked closely with our patient-research partner (SZ) in the design of the study who also aided in the search strategy. Our patient research partner aided in the clarification of research questions as well. The involvement of patient research partners allows for suggestions of additional references as well as the provision of insights beyond those in the literature. ${ }^{15}$

\section{RESULTS}

A total of 36643 citations were retrieved, and on duplicate removal, a total of 16173 citations were reviewed at the title and abstract stage for inclusion (figure 1). A total 184 full-text peer-reviewed articles were assessed for eligibility for inclusion; of these, 9 articles were included in this review (figure 1). From the non-peer-reviewed literature, following the title and document review, eight documents met the criteria for inclusion in this study (figure 1). The most common reason for 

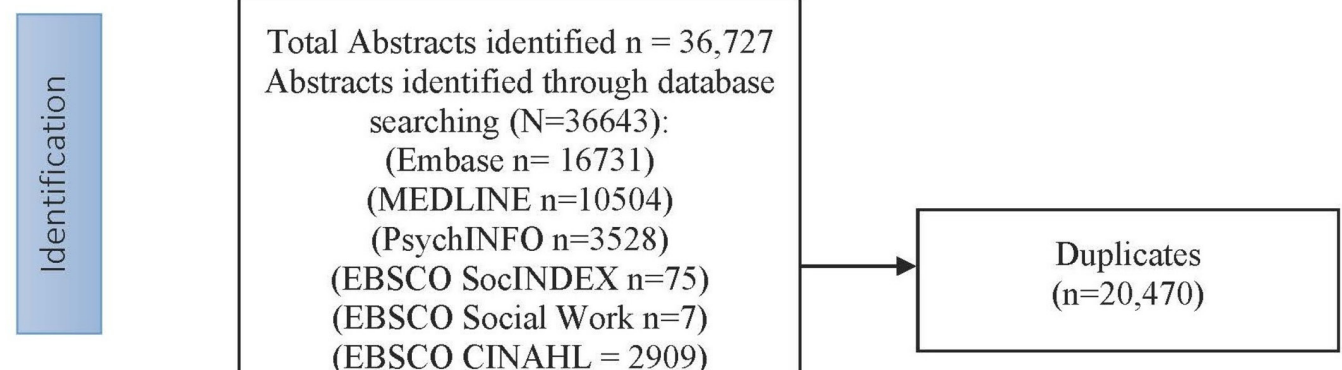

(Cochrane Central database $\mathrm{n}=2889$ )

Abstracts identified through Grey Literature $n=84$
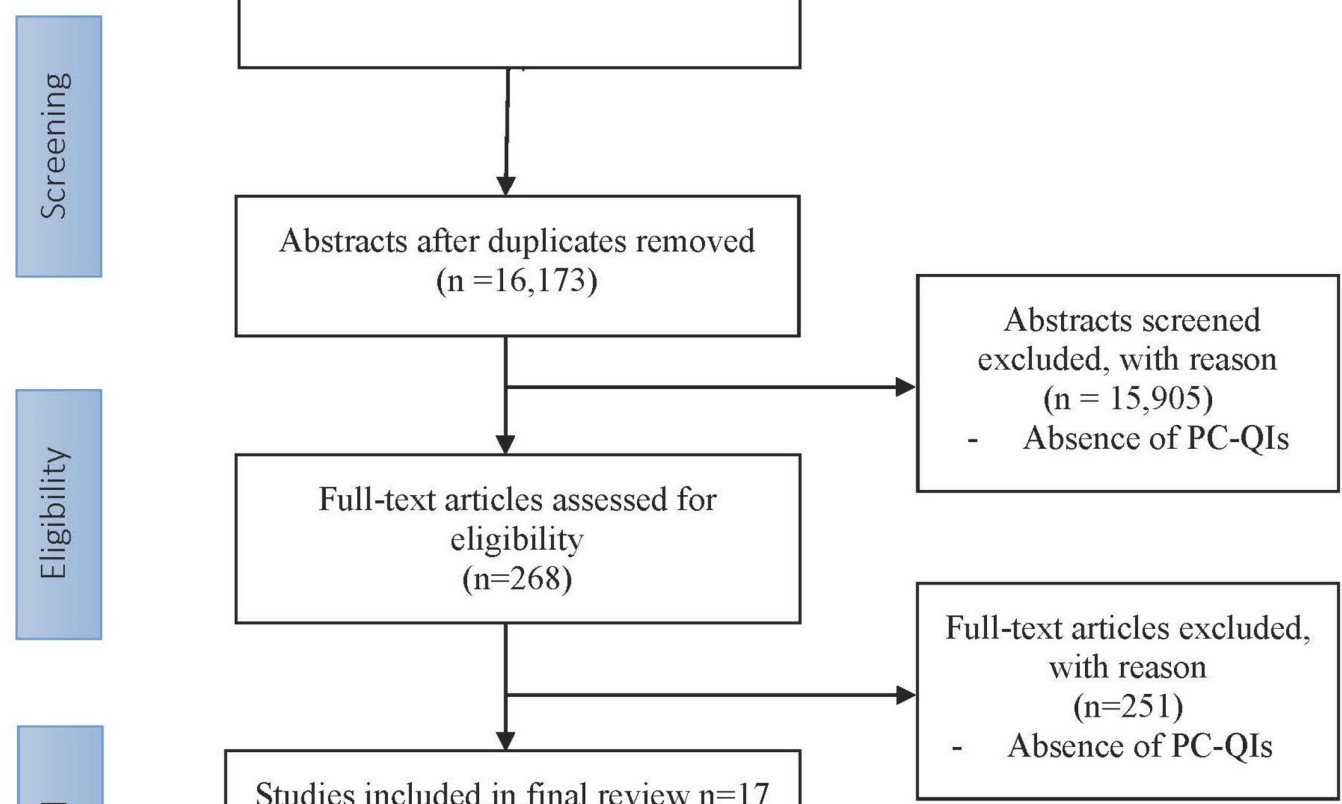

$n=9$ peer reviewed $\mathrm{n}=8$ grey literature

Figure 1 Flow diagram for study inclusion. PC-QIs, patient-centred quality indicators.

excluding articles $(\mathrm{n}=15905)$ was the absence of indicators for PCC.

\section{Article description}

Table 1 presents the characteristics of the full-text articles included in the study. The years of publication ranged from 1996 to 2015. Included studies were published in Belgium ( $n=1)$, USA $(n=2)$, Canada $(n=3)$, UK $(n=4)$, the Netherlands $(n=4)$ and three sources did not include a country of publication. Sources were varied and included original peer-reviewed research $(n=8)$, guest editorial $(n=1)$, reports $(n=4)$, discussion paper $(n=1)$, working paper $(n=1)$, literature review $(n=1)$ and a website $(n=1)$. The study populations varied in the peer-reviewed literature (eg, cancer, fertility care and home parenteral nutrition, HPN) and no specific populations were identified in the non-peer-reviewed literature.

What PC-QIs have been developed to measure patient centred care?

From the sources included, a total of 502 ways of measuring PCC were explicitly identified as quality indicators by studies' authors. However, only 25 were classified as actual indicators by our research team.

PCC measurement varied between articles. While all sources used the term 'quality indicator', not all were quantifiable and measurable. Most sources presented quality indicators as guidelines or recommendations for healthcare practitioners. Wensing et al presented indicators as survey items, such as 'Does the GP pay attention 


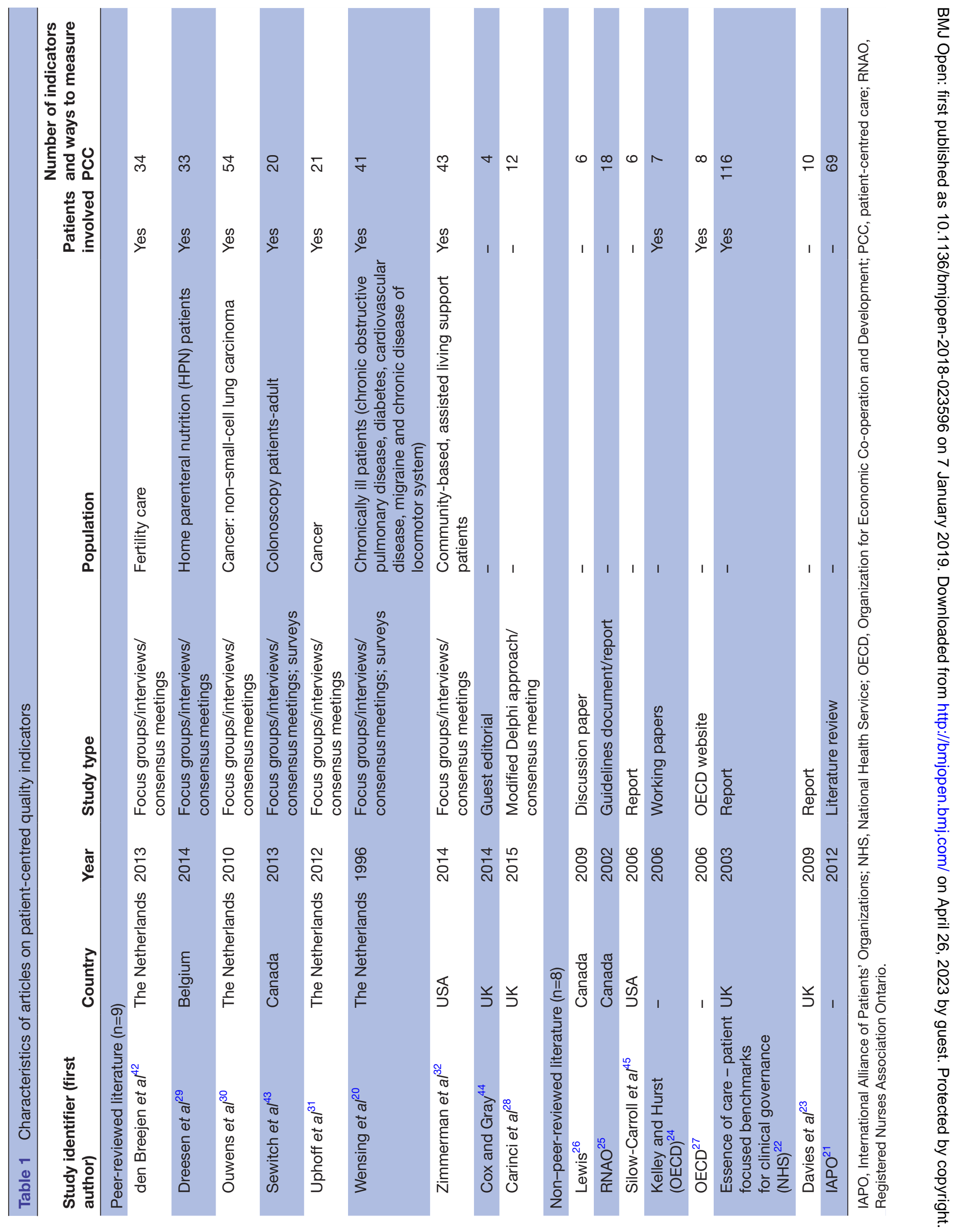


Table 2 Examples of patient-centred care (PCC) measurement classified according to the person-centred care framework ${ }^{18}$

\begin{tabular}{|c|c|c|}
\hline $\begin{array}{l}\text { PCC measure } \\
\text { classification }\end{array}$ & Domain & Example of ways to measure PCC \\
\hline Structure $(n=80)$ & $\begin{array}{l}\text { Supporting a workforce committed } \\
\text { to PCC. }\end{array}$ & $\begin{array}{l}\text { The development needs of healthcare personnel are met by ongoing } \\
\text { review through supervision, appraisal and individual development } \\
\text { plans. }{ }^{22} \\
\text { All staff and volunteers can articulate person-centred principles } \\
\text { and practices applicable to their role(s) and demonstrate their } \\
\text { implementation. }{ }^{32}\end{array}$ \\
\hline $\begin{array}{l}\text { Process } \\
(n=343)\end{array}$ & Cultivating communication. & $\begin{array}{l}\text { (Regular) doctor involving patients in decisions about care or } \\
\text { treatment. } \\
\text { Making use of open-ended questions in a conversation with the } \\
\text { patient. } \\
\text { Patient had the knowledge and support to make decisions. } \\
\text { Pegular doctor providing easy-to-understand explanations. } \\
\text { Pe }\end{array}$ \\
\hline $\begin{array}{l}\text { Outcome } \\
(n=79)\end{array}$ & Patient-reported outcomes. & $\begin{array}{l}\text { Measures are in place to assess and provide feedback on the } \\
\text { interpersonal skills of healthcare personnel. }{ }^{22} \\
\text { Regarding infertility treatment, patients would like to see all members } \\
\text { of the infertility treatment team following the same policy. }{ }^{42}\end{array}$ \\
\hline
\end{tabular}

to patients' needs?' ${ }^{20}$ Actual indicators were presented as percentages and proportions, and identified from six grey literature sources. ${ }^{21-26}$ For instance, one study outlined an example of a structure indicator- percent of nurses attending education sessions (orientation, organization professional development opportunities) on client-centred care'. 25

Study populations included cancer patients $(n=2)$, colonoscopy patients $(n=1)$, HPN patients $(n=1)$, chronically ill $(n=1)$, fertility care $(n=1)$ and assisted living support patients $(n=1)$ (table 1$)$. For the development of PCC measures, 7 studies used focus groups, interviews and/ or consensus meetings, 10 studies included patients and family caregivers in the development of PCC measures and 1 article developed measures through the authors' clinical and research work. Two sources developed ways to measure PCC from patient-reported experience surveys, ${ }^{24} 27$ and two studies used a framework. ${ }^{25} 28$

Some studies grouped ways to measure PCC according to domains of PCC that were based on previously defined frameworks or through consensus (eg, access to care, communication and information). Domains identified from the person-centred care framework are categorised according to structure, process and outcome. ${ }^{18}$ Here table 2 presents examples of measuring PCC classified according to the person-centred care framework, ${ }^{18}$ and table 3 presents the actual indicators classified according to the same framework. $^{18}$

\section{How are PC-Qls defined?}

The definition of a PC-QI was not clearly articulated in the studies identified (table 4). Ten of the included studies provided no such definition of a quality indicator (table 4). Sources where the definition of a quality indicator was mentioned defined indicators as something to be measured, and developed through consensus (table 4). ${ }^{2329-31}$ Of those four sources where the definition of a PC-QI was clear, two included actual indicators. ${ }^{21} 23$ The National Health Service report described indicators as items that patients, carers and professionals believed were important in achieving the benchmarks of best practice'. ${ }^{22}$

Have the existing PC-QIs been implemented and evaluated across various points-of-care settings, processes of care and at the system level to measure PCC?

None of the articles mentioned actual implementation of indicators in the settings for which they were developed. Similarly, many of the sources (15/17) did not evaluate the indicators according to any set criteria. The two studies that did evaluate indicators used two different approaches. $^{2830}$ The study by Ouwens et al assessed guidelines for psychometric characteristics and only 26 out of 56 guidelines were found to be reliable. ${ }^{30}$ The study by Carinci et al used a modified Delphi approach with expert Organization for Economic Co-operation and Development members to rate the PCC measures, using validity, reliability, relevance, actionability, international feasibility and international comparability as the criteria. ${ }^{28}$ Uphoff et al recommended using the Gol and Grimshaw model for evaluating indicators, ${ }^{31}$ while a future direction for Zimmerman et al was the evaluation of the indicators. ${ }^{32}$ The working paper by Kelley and Hurst presented criteria that can be used to select indicators. ${ }^{24}$ These included the importance of what is being measured (which includes 
Table 3 Identified PC-Qls from the literature classified according to the person-centred care framework as actual indicators ${ }^{18}$

\begin{tabular}{ll}
\hline $\begin{array}{l}\text { Type of } \\
\text { indicator }\end{array}$ & Domain \\
\hline Structure & Creating a PCC culture \\
& \\
& $\begin{array}{l}\text { Supporting a workforce committed } \\
\text { to PCC }\end{array}$
\end{tabular}

Process Cultivating communication

Respectful and compassionate care

Engaging patients in managing their care

Integration of care

Access to care

\section{PCC indicators $(n=25)$}

- An induction programme is in place which promotes the philosophy of care. ${ }^{22}$

- \% of PHC organisations who currently have processes to involve community input for planning the organisation's services (eg, advisory committees and focus groups). ${ }^{21}$

- Clear policies are in place on how services are offered to patients. ${ }^{21}$

- Percent of nurses attending education sessions (orientation and organisation professional development opportunities) on client-centred care. ${ }^{25}$

- Percent of non-nursing staff attending education sessions (orientation and organisation professional development opportunities) on client-centred care. ${ }^{25}$

- Percent of patients with access to an online HER (a) by region and (b) by practice. ${ }^{26}$

- Proportion of service users who stated that the district nurse provided health advice or information about his/her condition. ${ }^{23}$

- Proportion of service users who stated that they were involved as much as they wanted to be in decisions about their care and treatment. $^{23}$

- Proportion of service users who stated that their district nurse treated them with respect and dignity. ${ }^{23}$

- Percent of inpatients who said they were always treated with respect and dignity while in hospital/primary care. ${ }^{21}$

- Percent of PHC clients/patients, 18years and older, with a chronic condition(s), who actively participated in the development of a treatment plan with their PHC provider over the past 12 months. $^{21}$

- Percent of hospital patients who said they had been sufficiently involved in decisions about their care as much as they wanted to be. ${ }^{21}$

- Percent of nurses self-reporting: adequate assessment of a client's perceived needs for care, adequate assessment of a client's goals for care, adequate documentation of a client's personal goals for care, sharing client's concerns/choices with other members of the healthcare team, discharge teaching guided by the client's goals for managing their care at home. ${ }^{25}$

- Percent of hospital patients taking medicines home after discharge who were told completely about the purposes of the medicine in a way they could understand. ${ }^{21}$

- Percentage of patients who can get all diagnostic work ordered by their primary care doctor done the same day in the same location (excluding certain high-technology procedures such as CT and $\mathrm{MRI})^{26}$

- Percentage of out-patients seen within 13 weeks of GP referral. ${ }^{24}$

- Percentage of those on waiting list waiting 12 months or more. ${ }^{24}$

- Proportion of service users who were able to contact a district nurse when needed, including outside of normal working hours. ${ }^{23}$

- The percentage of patients who, in the appropriate national survey, indicate that they were able to obtain a consultation with a GP or appropriate healthcare professional within two working days (NHS Confederation, UK). ${ }^{21}$

- Percentage of PHC clients/patients, 18years and older, with a chronic condition(s), who had sufficient time in most visits to confide their health-related feelings, fears and concerns to their PHC provider. ${ }^{21}$ 


\begin{tabular}{|c|c|c|}
\hline $\begin{array}{l}\text { Type of } \\
\text { indicator }\end{array}$ & Domain & PCC indicators $(n=25)$ \\
\hline Outcome & Patient-reported experiences & $\begin{array}{l}\text { Proportion of service users who stated that the district nurse } \\
\text { had all the necessary information about the service user and his/ } \\
\text { herhealth needs. } \\
\text { Proportion of service users who stated that the district nurse had } \\
\text { all the equipment and dressings needed. } \\
\text { Proportion of service users who stated that the district nurse was } \\
\text { knowledgeable and competent. }^{23} \\
\text { Proportion of service users who rated the district nurse service as } \\
\text { very good or excellent. } \\
\text { Proportion of children whose parents routinely received all aspects } \\
\text { of family-centred care (child and adolescent health measurement } \\
\text { initiative). } .^{21}\end{array}$ \\
\hline
\end{tabular}

GP, general practitioner; HER, health electronic record; NHS, National Health Service; PCC, patient-centred care; PC-QIs, patient-centred quality indicators; $\mathrm{PHC}$, primary healthcare.

policy importance), scientific soundness and feasibility of the measure. ${ }^{24}$

\section{DISCUSSION}

This review specifically examined existing PC-QIs in the academic and grey literature. PCC has been increasingly adopted by many jurisdictions; however, this review revealed there to be gaps in the conceptualisation of PC-QIs. Out of the 17 articles that met the inclusion criteria, only 9 were peer reviewed, and looked at specific conditions. Additionally, the heterogeneity of the PCC literature relates to the variety of definitions on PC-QIs, and a diverse type of indicators developed for different patient population and care-settings. For instance, all included articles in the review used the term 'indicator,' but not all presented actual indicators as defined by the AHRQ. ${ }^{16}$

The absence of a standard definition of what a PC-QI is in the literature has posed challenges in identifying the literature sources for inclusion in this review. 'Quality' means different things to different people, and despite the standard definition of PCC by the

Table 4 Definition of quality indicator used by authors

\section{Study identifier (first author, year)}

den Breejen et al (2013), ${ }^{42}$ Sewitch et al (2013), ${ }^{43}$ Uphoff et al (2012), ${ }^{31}$ Zimmerman et al (2014), ${ }^{32}$ Cox and Gray (2015), ${ }^{44}$ Lewis (2009), ${ }^{26}$ RNAO (2002), ${ }^{25}$ Silow-Carroll et al (2006), ${ }^{45}$ Kelley and Hurst (2006), ${ }^{24}$ OECD website $(2006)^{27}$ and Carinci et al $\left(2015^{28}\right.$

Dreesen et al $(2014)^{29}$

Ouwens et al (2010) ${ }^{30}$ and Uphoff et al (2012) ${ }^{31}$

Davies et al $(2011)^{23}$

NHS $(2003)^{22}$

IAPO $(2012)^{21}$

\section{Definition of quality indicator used}

Definition not included.

A quality indicator as a measurable element of practice performance for which there is evidence or consensus that it can be used to assess the quality of care, and hence change the care provided. ${ }^{46}$

Quality indicators are 'measurable elements of practice performance for which there is evidence or consensus that they can be used to assess the quality of care'. ${ }^{47}$

An explicit measurable statement of the quality of care given. Relates to a single outcome or process of medical care. Clearly defined and unambiguous. ${ }^{47} 48$

Items that patients, caregivers and professionals believed were important in achieving the benchmarks of best practice. $^{22}$

Can be measures of structure, process and outcome, either as generic measures relevant for all diseases, or diseasespecific measures that describe quality of patient care related to a specific diagnosis. ${ }^{49}$ 
Institute of Medicine, ${ }^{1}$ PCC continues to be operationalised and measured differently. ${ }^{33}$ Most sources identified in the literature did not explicitly define what a quality indicator is, which may explain the differences in approaches to indicator development. The absence of a definition for quality indicators results in inconsistencies for how an indicator should be presented and also what makes for a good indicator. Previous reviews have also found variability in quality indicator definitions, such as indicators for hip fracture patient care. ${ }^{34}$ The absence of a standard definition of PC-QIs poses concerns for standardised measurement of PCC, and for implementation of PC-QIs in healthcare settings.

In compliance with quality improvement agencies, quality councils and organisations such as the AHRQ, quality indicators should be presented as a unit of measurement-as a percentage or proportion. ${ }^{16}$ Our review revealed inconsistencies in the definition of indicators. Only few sources in the non-peer-reviewed literature included PC-QIs as quantifiable units, such as percentages, incorporating a numerator and denominator in the unit of measurement. ${ }^{35}$ Most of the identified indicators were actually domains included in PCC measures and guidelines. The lack of defined units of measurement impedes comparisons across facilities, and benchmarking, and does not allow for longitudinal evaluation and overall measurement of care that is patient-centred. Without this unit of measurement for PC-QIs, it is difficult to target specific improvements needed for PCC.

The review revealed that when incorporating the patient and caregiver perspective in quality improvement, a difficulty exists in translating perceptions and subjective experiences into standardised objective indicators. Measures of well-being are both necessary and important to incorporating a PCC model of care. The study by Carr et alsuggests that perceptions of health and its meaning vary between individuals and across time, as do their experiences and expectations of healthcare. ${ }^{36}$ In order to capture various perspectives on quality care, it is vital to include patients and families. Including the patient and family perspective is necessary to ensure quality PCC.

Additionally, this review found large variances for domains to categorise approaches to measuring PCC. In an attempt to organise our findings and understand the ways of measuring PCC, we used a previously published person-centred care framework ${ }^{18}$ to classify them into healthcare quality domains. From this classification, most strategies for measuring PCC were found to relate to domains associated with healthcare processes (eg, cultivating communication). These findings are consistent with the current measurement landscape, for instance trauma indicators, ${ }^{37}$ and AHRQ PC-QIs ${ }^{38}$ which mainly assess processes and outcomes. For PCC, structures, such as policies and education programmes can provide an important basis to improve PCC practice. ${ }^{18} 39$ Structure indicators provide the necessary foundations for the assessment of process and outcome indicators, for instance through creating a PCC culture, supporting the workforce to deliver PCC and providing an accommodating environment for patients. ${ }^{18}$ In this review, structure indicators were lacking.

Finally, there is scarce evidence in the literature on how to implement indicators for PCC, and how to evaluate their implementation. PCC measurement has, to date, primarily focused on specific disease conditions and healthcare sectors. However, recent initiatives (National Health Service) reveal a more generic approach to measurement (Family and Friend Test). ${ }^{40}$ In order to create a standardised set of PC-QIs, indicators must be developed across the continuum of care. Santana and Stelfox also found a lack of implementation of indicators in care settings in their review. ${ }^{37}$ Before indicators are implemented, they must be evaluated according to standard set criteria. What constitutes as a good indicator has been outlined by health quality organisations, such as National Quality Forum. As outlined by the National Quality Forum, quality indicators should be evaluated through a set criterion including importance, scientific acceptability, feasibility, and usability and use. ${ }^{41}$ Our review did not identify any studies where such evaluations were implemented. In the development and implementation of quality indicators, the guideline set by the National Quality Forum should be adhered to.

The gaps identified in the literature for PC-QIs provide directions for future research. First, there needs to be consensus on a standard definition of PC-QI to guide future measurement of PCC. Second, there is a need to develop a standard set of PC-QIs that could be implemented in various healthcare settings. Third, PC-QIs need to be evaluated according to a set criteria. Finally, PC-QIs need to be implemented across healthcare settings for monitoring and evaluation of PCC.

\section{Strengths and limitations}

Our scoping review used robust and transparent methods guided by a protocol previously published, ${ }^{13}$ and supported by a research librarian with expertise in knowledge synthesis and scoping reviews (DLL). We worked closely with our patient research partner (SZ) in the study design, research questions, search strategy and manuscript preparation.

This review may not have identified all relevant sources in the published and grey literature as we conducted the search using only English terms. As we are following the scoping review methodology, the quality of the studies was not assessed.

\section{CONCLUSIONS}

In summary, our review is the first to examine the literature pertaining to quality indicators that are patient-centric. Our findings will further the development of 
validated healthcare tools assessing healthcare quality from a patient-centred approach. Future research should focus on developing and refining PC-QIs that are ready to implement and evaluate following the criteria set forth by the National Quality Forum. ${ }^{41}$

Acknowledgements We would like to acknowledge the contributions of Lisa Goodman, who provided support for the grey literature search for this scoping review.

Contributors M-JS conceived the study, and all authors identified key literature to be included in the review. M-JS led the drafting of the manuscript and key discussion points with support from DL, RJJ, SA, ML and KM. SA managed the design of the tables (with feedback from all authors), and management of references. All authors provided important intellectual contribution and guidance throughout the development of the manuscript. HQ, DL, SZ and ML provided guidance on the presentation of the findings and guidance on final revisions. All of the authors contributed to critical review and revisions to the manuscript, agreeing on the final version.

Funding M-JS secured funding from the M.S.I Foundation (Grant \#886) based in Alberta, Canada. ML acknowledges the support from the Alberta's Strategy for Patient Oriented Research (SPOR).

Competing interests None declared.

Patient consent for publication Not required.

Provenance and peer review Not commissioned; externally peer reviewed.

Data sharing statement № additional data available.

Open access This is an open access article distributed in accordance with the Creative Commons Attribution Non Commercial (CC BY-NC 4.0) license, which permits others to distribute, remix, adapt, build upon this work non-commercially, and license their derivative works on different terms, provided the original work is properly cited, appropriate credit is given, any changes made indicated, and the use is non-commercial. See: http://creativecommons.org/licenses/by-nc/4.0/.

\section{REFERENCES}

1. Institute of Medicine. Crossing the quality chasm: a new health system for the 21st century: National Academy Press, 2001.

2. Doyle C, Lennox L, Bell D. A systematic review of evidence on the links between patient experience and clinical safety and effectiveness. BMJ Open 2013;3:e001570.

3. Oates J, Weston WW, Jordan J. The impact of patient-centered care on outcomes. Fam Pract 2000;49:796-804.

4. Fung $\mathrm{CH}$, Lim Y-W, Mattke S, et al. Systematic review: the evidence that publishing patient care performance data improves quality of care. Ann Intern Med 2008;148:111-23.

5. Epstein RM, Fiscella K, Lesser CS, et al. Why the nation needs a policy push on patient-centered health care. Health Aff 2010;29:1489-95.

6. Bertakis KD, Azari R. Patient-centered care is associated with decreased health care utilization. J Am Board Fam Med 2011;24:229-39.

7. Delaney LJ. Patient-centred care as an approach to improving health care in Australia. Collegian 2017;25:119 123.

8. Fix GM, VanDeusen Lukas C, Bolton RE, et al. Patient-centred care is a way of doing things: How healthcare employees conceptualize patient-centred care. Health Expect 2018;21:300-7.

9. Dwamena F, Holmes-Rovner M, Gaulden CM, et al. Interventions for providers to promote a patient-centred approach in clinical consultations. Cochrane Database Syst Rev 2012;12:CD003267.

10. Braithwaite J, Hibbert P, Blakely B, et al. Health system frameworks and performance indicators in eight countries: a comparative international analysis. SAGE Open Med 2017;5:20.

11. Agency for Healthcare Research and Quality. Quality indicator measure development, implementation, maintenance, and retirement. Rockville, MD: Agency for Healthcare Research and Quality: Center for Health Policy Center for Primary Care and Outcomes Research \& Battelle Memorial Institute, 2011.

12. Hughes RG. Tools and strategies for quality improvement and patient safety. 2008

13. Jolley RJ, Lorenzetti DL, Manalili K, et al. Protocol for a scoping review study to identify and classify patient-centred quality indicators. BMJ Open 2017;7:e013632.
14. Arksey H, O'Malley L. Scoping studies: towards a methodological framework. Int J Soc Res Methodol 2005;8:19-32.

15. Levac D, Colquhoun H, O'Brien KK. Scoping studies: advancing the methodology. Implement Sci 2010;5:69.

16. Agency for Healthcare Research and Quality. Quality Improvement and monitoring at your fingertips. Secondary Quality Improvement and monitoring at your fingertips. https://www.qualityindicators.ahrq gov/ (Accessed 01 Oct 2017).

17. Synthesis. Secondary Synthesis. 2016 http://synthesis.info/ (Accessed 01 Oct 2017)

18. Santana MJ, Manalili K, Jolley RJ, et al. How to practice personcentred care: a conceptual framework. Health Expect 2018;21.

19. Donabedian A. Quality assessment and monitoring. Retrospect and prospect. Eval Health Prof 1983;6:363-75.

20. Wensing M, Grol R, van Montfort P, et al. Indicators of the quality of general practice care of patients with chronic illness: a step towards the real involvement of patients in the assessment of the quality of care. Qual Health Care 1996;5:73-80.

21. International Alliance of Patients' Organizations(IAPO). Patientcentred healthcare indicators review. 2012 http://iapo.org.uk/ sites/default/files/files/IAPO\%2OPatient-Centred\%20Healthcare\% 20Indicators\%20Review.pdf.

22. NHS Dignity Care. Essence of Care Patient-focused benchmarks for clinical governance. 2003 https://www.dignityincare.org.uk/_assets/ Resources/Dignity/OtherOrganisation/Essence of Care.pdf.

23. Davies P, Wye L, Horrocks S, et al. Developing quality indicators for community services: the case of district nursing. Qual Prim Care 2011:19:155.

24. Kelley E, Hurst J. Health care quality indicators project. $O E C D$ HEALTH WORKING PAPERS NO. 232006 https://www.oecd.org/els/ health-systems/36262363.pdf.

25. Registered Nurses Association of Ontario. Client centred care. Toronto, Ontario, 2002

26. Lewis S. Patient-centered care: an introduction to what it is and how to achieve it. Saskatchewan: Ministry of Health, 2009.

27. OECD. Health care quality indicators - responsiveness and patient experiences. Secondary health care quality indicators responsiveness and patient experiences. 2006. http://www.oecd.org/ els/health-systems/hcqi-responsiveness-and-patient-experiences. htm (Accessed 01 Oct 2017).

28. Carinci F, Van Gool K, Mainz J, et al. Towards actionable international comparisons of health system performance: expert revision of the OECD framework and quality indicators. Int $J$ Qual Health Care 2015;27:137-46.

29. Dreesen M, Foulon V, Vanhaecht K, et al. Identifying patient-centered quality indicators for the care of adult home parenteral nutrition (HPN) patients. JPEN J Parenter Enteral Nutr 2014;38:840-6.

30. Ouwens M, Hermens R, Hulscher M, et al. Development of indicators for patient-centred cancer care. Support Care Cancer 2010;18:121-30.

31. Uphoff EP, Wennekes L, Punt CJ, et al. Development of generic quality indicators for patient-centered cancer care by using a RAND modified Delphi method. Cancer Nurs 2012;35:29-37.

32. Zimmerman S, Love K, Cohen LW, et al. Person-centeredness in home- and community-based services and supports: domains, attributes, and assisted living indicators. Clin Gerontol 2014:37:429-45.

33. Epstein RM, Franks P, Fiscella K, et al. Measuring patient-centered communication in patient-physician consultations: theoretical and practical issues. Soc Sci Med 2005;61:1516-28.

34. Pitzul KB, Munce SE, Perrier L, et al. Scoping review of potential quality indicators for hip fracture patient care. BMJ Open 2017;7:e014769.

35. Hermann RC, Mattke S, Somekh D, et al. Quality indicators for international benchmarking of mental health care. Int J Qual Health Care 2006;18:31-8.

36. Carr AJ, Gibson B, Robinson PG. Measuring quality of life: Is quality of life determined by expectations or experience? BMJ $2001 ; 322: 1240-3$

37. Santana MJ, Stelfox HT. Quality indicators used by trauma centers for performance measurement. J Trauma Acute Care Surg 2012;72:1298-303.

38. Agency for Healthcare Research and Quality. Pediatric quality indicators overview. secondary pediatric quality indicators overview 2017. https://www.qualityindicators.ahrq.gov/modules/ pdi_resources.aspx (Accessed 01 Oct 2017).

39. The Lewin Group. Indicators of cultural competence in health care delivery organizations: an organizational cultural competence assessment profile. $2002 \mathrm{https}: / /$ www.hrsa.gov/sites

40. National Health Services UK. Friends and family test 2017. https:// www.england.nhs.uk/fft/ (accessed 20 Dec 2017). 
41. National Quality Forum. Measure evaluation criteria. Secondary measure evaluation criteria. http://www.qualityforum.org/ Measuring_Performance/Submitting_Standards/Measure_ Evaluation_Criteria.aspx (Accessed 01 Oct 2017).

42. den Breejen EM, Nelen WL, Schol SF, et al. Development of guideline-based indicators for patient-centredness in fertility care: what patients add. Hum Reprod 2013;28:987-96.

43. Sewitch MJ, Dubé C, Brien S, et al. Patient-identified quality indicators for colonoscopy services. Can J Gastroenterol 2013;27:25-32.

44. Cox J, Gray A. NHS at the hustings: four quality indicators for a person centred medicine. Eur J Pers Cent Healthc 2015;3:1-3.
45. Silow-Carroll S, Alteras T, Stepnick L. Patient-centered care for underserved populations: definition and best practices. Washington, DC: Economic and Social Research Institute, 2006.

46. Lawrence M, Olesen F. Indicators of quality in health care. Eur J Gen Pract 1997;3:103-8.

47. Campbell SM, Braspenning J, Hutchinson A, et al. Research methods used in developing and applying quality indicators in primary care. Qual Saf Health Care 2002;11:358-64.

48. Pencheon D. The good indicators guide: understanding how to use and choose indicators: NHS Institute for Innovation and Improvement, 2008.

49. Mainz J. Defining and classifying clinical indicators for quality improvement. Int J Qual Health Care 2003;15:523-30. 